\title{
Natural Antioxidant Activity and Compounds Content from Wastes of Euterpe edulis Berries
}

\author{
Beatriz da Silva Frasao ${ }^{1,2}$, Marion Pereira da Costa ${ }^{1,3}$, Bruna Leal Rodrigues ${ }^{1}$, Bruno Reis Costa Lima ${ }^{1}$ \\ \& Carlos Adam Conte-Junior ${ }^{1,3}$ \\ ${ }^{1}$ Department of Food Technology, Faculdade de Veterinária, Universidade Federal Fluminense, Rio de Janeiro, \\ Brazil \\ ${ }^{2}$ Department of Epidemiology and Public Health, Instituto de Veterinária, Universidade Federal Rural do Rio de \\ Janeiro, Rio de Janeiro, Brazil \\ ${ }^{3}$ Food Science Program, Instituto de Química, Universidade Federal do Rio de Janeiro, Rio de Janeiro, Brazil \\ Correspondence: Marion Pereira da Costa, Department of Food Technology, Faculdade de Veterinária, \\ Universidade Federal Fluminense, Rua Vital Brazil Filho, n. 64. Santa Rosa, 24230-340, Rio de Janeiro, Brazil. \\ Tel: 55-21-2629-9545. E-mail: marioncosta@id.uff.br
}

\begin{tabular}{|c|c|c|}
\hline 16 & Accepted: January 20, 2017 & Online Published: February 15, 2017 \\
\hline loi:10.5539/jas. & $\mathrm{g} / 1055$ & n $3 \mathrm{n} 17$ \\
\hline
\end{tabular}

The research is financed by Juçai Industry (Juçai ${ }^{\circledR}$, Rio de Janeiro, Brazil); Fundação de Amparo à Pesquisa do Estado do Rio de Janeiro (processes E-26/201.185/2014 and E-26/010.001.911/2015, FAPERJ, Brazil); Conselho Nacional de Dessenvolvimento Científico e Tecnológico (process 311361/2013-7 and 400136/2014-7, CNPq, Brazil); Coordenação de Aperfeiçoamento de Pessoal de Nivel Superior (process 125, CAPES/Embrapa 2014, CAPES, Brazil).

\begin{abstract}
The Euterpe edulis (Juçara) is native to Brazil, which berries and wastes present high antioxidant content. Therefore, in this study, microwave-assisted extraction (MAE) was investigated for antioxidant compounds extraction from E. edulis waste and maximized antioxidant activities using response surface methodology coupled with a central composite design. Three factors were observed: microwave power $(400 / 500 / 600 \mathrm{~W})$, exposition time $(30 / 60 / 90 \mathrm{sec})$ and ethanol concentration solvent $(40 / 60 / 80 \%)$. The extracts were characterized by determination of total phenolic (TPC), flavonoids (TFC), monomeric anthocyanins (TAC), tannins content (TTC), and in vitro antioxidant assay (AA\%). The yield of TPC, TFC, TAC, and TTC varied at 595.43-2171.34 mg GAE·100 g DM$^{-1}, 137.36-251.24 \mathrm{mg}$ QE·100 $\mathrm{g} \mathrm{DM}^{-1}$, 179.32-354.38 mg C-3-GE·100 g DM ${ }^{-1}$ and 0.23-1.00 $\mu \mathrm{g}$ TAE $100 \mathrm{~g} \mathrm{DM}^{-1}$, respectively. The optimal MAE parameters for TPC was microwave power $668.18 \mathrm{~W}$, exposition time $110.45 \mathrm{~s}$ and aqueous ethanol concentration $93.64 \%$, for TFC same parameters observed; though for TAC the different parameters were $532.28 \mathrm{~W}$, and for TTC $9.55 \mathrm{~s}$. However, for antioxidant activity, the parameters were $668.18 \mathrm{~W}, 110.45 \mathrm{~s}$ time and $64.41 \%$ of aqueous ethanol solvent. Therefore, this methodology was successfully applied for optimal extraction of total phenolics, flavonoids, monomeric anthocyanins and tannins from juçara waste and obtain optimal antioxidant activity.
\end{abstract}

Keywords: anthocyanin, $\beta$-carotene bleaching, flavonoid, microwave-assistant extraction, phenolic, RSM optimization, tannin

\section{Introduction}

The Juçara palm (Euterpe edulis Martius; Arecaceae), a native plant of Brazilian Atlantic Forest, is widely distributed in Brazil. Mature berries from this plant exhibit a violaceous and globose shape, which are accepted by consumers (Silva et al., 2013). Juçara fruit is commercialized in the form of pulp or juice. During the processing of E. edulis pulp, the seeds with endocarp and epicarp are discarded as waste (Bicudo, Ribani, \& Beta, 2014). The violaceous color of E. edulis pulp can be related to the presence of anthocyanins, which belong to the group of flavonoids (Cavalcanti, Santos, \& Meireles, 2011). In addition, other bioactive molecules including phenolic acids, flavonoids, and tannins were also identified in juçara berries (Bicudo et al., 2014; Borges et al., 2013; Da Silva Campelo Borges et al., 2011; Rufino, Alves, Fernandes, \& Brito, 2011), which is associated with 
high antioxidant capacity (Rufino et al., 2011). In addition, Garcia-Mendoza et al (Garcia-Mendoza et al., 2017) demonstrate that industrial residue of juçara presented high concentration of phenolic acids and anthocyanins. In recent years, the use of plant extracts, mainly extract of their waste processing, have gained notable interest in the food industry (Ertas et al., 2015). This fact is related to the search for new therapeutic and preventive agents, as natural antioxidants, for amendments and illness, Alzheimer's disease, and cancer (Zengin, Sarikurkcu, Aktumsek, \& Ceylan, 2014).

Natural antioxidants agents present high attention in recent years for their bioactivity and safety (Lu, Qin, Han, Wang, \& Li, 2015). The ingestion of this compounds is stimulated by potential neutralization effect on the toxicity of oxidative processes or prevention of prooxidant formation during digestion (Manganaris, Goulas, Vicente, \& Terry, 2014; Rahal et al., 2014), which can contribute to reducing or prevent the aforementioned illness. Furthermore, fruits with high antioxidant capacity, such as juçara (Borges et al., 2013) are potential source of bioactive molecules that can be a technological alternative for food industry to prevent the oxidation, providing an increased in food shelf life (Manganaris et al., 2014; Ortega-Ramirez et al., 2014; Tadapaneni, Daryaei, Krishnamurthy, Edirisinghe, \& Burton-Freeman, 2014). Therefore, the use of natural antioxidants capable of hindering oxidative processes responsible for losses in the organoleptic characteristics and the nutritional value of food is highly relevant for the food industry (Contini et al., 2014). However, the extraction of natural antioxidants compounds is still critical (Santos, Veggi, \& Meireles, 2010), where the evaluation of efficacy and efficiency of each extraction method is extremely important.

Several studies evaluated the influence of the extraction method on different matrices for the isolation of antioxidant compounds (Da Silva Campelo Borges et al., 2011; Dairi et al., 2015; Espinosa-Pardo, Martinez, \& Martinez-Correa, 2014; Kukula-Koch et al., 2013; Li, Ngadi, \& Ma, 2014). Nonetheless, different approaches and applications do not always provide the same results. Hence, the optimization processing is required (Santos et al., 2010). The optimum combination of power, exposition time, and concentration of extracting solvent to obtain the highest concentration of these compounds is extremely important to ensure efficient utilization of energy, solvents, and food matrix. In this way, microwave-assisted extraction (MAE), an emerging green technology, have demonstrated to be a promising method for the recovery of bioactive compounds such as total phenolics, flavonoids, anthocyanins, and tannins from plants. (Dahmoune et al., 2014; Dahmoune, Nayak, Moussi, Remini, \& Madani, 2015; Dairi et al., 2015; Kim et al., 2012; Li et al., 2012, 2014; Zeković, Vladić, Vidović, Adamović, \& Pavlić, 2016). Although, to the best of our knowledge the MAE has not been used for extraction of natural antioxidant compounds from $E$. edulis.

Moreover, response surface methodology (RSM) can be used to optimize the extraction of natural antioxidant. The RSM is the combination of statistical and mathematical techniques which allow the improvement and optimization of processes. In this methodology, the response of interest is influenced by the independent variables or factors (Montgomery, 2004). Previous studies documented that the type of solvent, the temperature of extraction, exposition time, solid to liquid ratio, and microwave power influence the extraction of antioxidant molecules from fruits by MAE (Borges et al., 2011; Dairi et al., 2015). In this context, the aim of the present study was to utilize the antioxidants (total phenolic, flavonoids, anthocyanins, and tannins) present in the waste of pulp juçara processing. Besides, optimize the extraction of these compounds by MAE on the solid waste obtained during juçara berry processing applying RSM. Thereunto, three independent variables, microwave power (W), time exposition (s), and concentration of ethanol (\%), were evaluated.

\section{Materials and Methods}

\subsection{Chemicals and Reagents}

Ethyl alcohol 200 Proof $\left(\mathrm{C}_{3} \mathrm{H}_{3} \mathrm{C}_{2} \mathrm{H}_{2} \mathrm{OH}\right.$; cat. ER0515-002) was purchased from Tedia Brasil (Rio de Janeiro, RJ, Brazil); gallic acid (cat. 398225), tannic acid (cat. 403040), quercetin (cat. 4951), Folin-Ciocalteu's phenol reagent (F9252), aluminum chloride $\left(\mathrm{AlCl}_{3}\right.$; cat. 06220), poly(vinylpolypyrrolidone) (PVPP; cat. 77627), potassium chloride $(\mathrm{KCl}$; cat. 60130$)$, sodium acetate $\left(\mathrm{CH}_{3} \mathrm{COONa} \cdot 3 \mathrm{H}_{2} \mathrm{O}\right.$; cat. 32318), and potassium acetate $\left(\mathrm{CH}_{3} \mathrm{COOK}\right.$; cat. 236497) were purchased from Sigma Chemical Co. (St. Louis, MO, USA). Whereas, sodium carbonate $\left(\mathrm{Na}_{2} \mathrm{CO}_{3}\right)$ was purchased from Reagen (Rio de Janeiro, RJ, Brazil).

\subsection{Plant Material}

Juçara (Euterpe edulis) berries wastes (epicarp and endocarp) were supplied by Juçaí Industry (Juçaí ${ }^{\circledR}$, Rio de Janeiro, Brazil; $22^{\circ} 24^{\prime} 44^{\prime \prime} \mathrm{S}, 42^{\circ} 57^{\prime} 56^{\prime \prime} \mathrm{W}$ ) in May 2015. Wates were air dried at $24^{\circ} \mathrm{C}$ until constant weight (48 h), and then ground utilizing a manual burr grinder MSS-1B (Hario, Tokyo, Japan). Ground samples were sieved through a 250 Mesh screen as particle size affects the extractability of bioactive molecules (Shao et al., 2014). All samples were stored at $-20{ }^{\circ} \mathrm{C}$ until further analysis. 


\subsection{Experimental Design}

The optimal extractions conditions can be obtained by the ratio of responses based on variables in the process through the Response surface methodology (RSM) (Karacabey \& Mazza, 2010). A central composite design was utilized to determine the optimized condition in MAE extraction of total phenolic content (TPC), total flavonoids content (TFC), total monomeric anthocyanin content (TAC), total tannins content (TTC) and antioxidant activity from the juçara waste; non-coded and coded factors (microwave power, exposition time, and solvent concentration) are exhibited in Table 1. Although several factors such as microwave power, microwave temperature, exposure time, composition of solvent, solids to solid ratio and extraction cycles, can affect the extraction efficiency in MAE, studies show as the main independent variables microwave power, exposure time and solvent concentration ( $\mathrm{Li}$ et al., 2012, 2013; Zeković et al., 2016). To predict the optimal conditions of the extraction process experimental design software (Minitab ${ }^{\circledR} 17.1 .0$, USA) package was used for the regression analysis of the data to fit a second-order polynomial equation (Equation 1) for the regression analysis of the data.

$$
Y=\beta_{0}+\sum_{i=1}^{3} \beta_{i} \chi_{i}+\sum_{i=1}^{3} \beta_{i i} \chi_{i}^{2}+\sum_{i=1}^{2} \sum_{j=i+1}^{3} \beta_{i j} \chi_{i} \chi_{j}
$$

Where, TPC, TFC, TAC, and TTC values denote $Y_{1}, Y_{2}, Y_{3}$, and $Y_{4}$, respectively; whereas the three independent variables (or factors) were microwave power $\left(X_{1}\right)$, exposition time $\left(X_{2}\right)$, and ethanol concentration $\left(X_{3}\right) . \beta_{0}$ is the model constant, $\beta_{i}$ is the linear coefficient, $\beta_{\mathrm{ii}}$ is the quadratic coefficient, $\beta_{i j}$ is the two factors interaction coefficient, and $X_{i}$ and $X_{j}$ are independent variables (factors) level. According to the analysis of variance (ANOVA), the regression coefficients of individual linear, quadratic and interaction terms were determined.

\subsection{Microwave-Assisted Extraction (MAE)}

MAE present advantages compared with conventional extraction, which is considered a green technology (Zeković et al., 2016). Powder of Juçara waste was subjected to MAE utilizing a DGT 100 Plus system (Provecto Analytics Ltd., Jundiaí, SP, Brazil) for antioxidant extraction. Briefly, $500 \mathrm{mg}$ of waste were added to $25 \mathrm{~mL}$ of aqueous ethanol solution, sealed into the extraction vessels, and subjected to extraction protocol following the experimental design (Table 1). After each extraction, the vessels were centrifuged at $1,400 \times \mathrm{g}$ for 10 min at $4{ }^{\circ} \mathrm{C}$ and cooled to $25{ }^{\circ} \mathrm{C}$. The precipitate was re-extracted with an additional $25 \mathrm{~mL}$ of the same ethanol solution and at the same MAE conditions; the supernatants were pooled and stored in amber vials at 4 ${ }^{\circ} \mathrm{C}$.

\subsection{Determination of Total Phenolic Content (TPC)}

TPC of the extracts of E. edulis waste was estimated based on Folin-Ciocalteu method described by Ainsworth \& Gillespie (2007). The absorbance value at $765 \mathrm{~nm}$ was recorded using a Spectrophotometer UV-1800 (Shimadzu Corporation, Kyoto, Japan) and the results were calculated based on a calibration curve of gallic acid (0.00-1.25 $\mathrm{mg} \mathrm{L}^{-1}$ ). The results were expressed as $\mathrm{mg}$ gallic acid equivalent (GAE) per $100 \mathrm{~g}$ of dry matter (DM).

\subsection{Determination of Total Flavonoids Content (TFC)}

The TFC extracts of E. edulis waste were estimated by a colorimetric method developed by Chang, Yang, Wen, and Chern (2002) utilizing aluminum chloride. The absorbance was read at 415 and $700 \mathrm{~nm}$ using Spectrophotometer UV-1800 (Shimadzu Corporation, Kyoto, Japan); the latter wavelength was utilized to correct the influence of haze. A calibration curve utilizing quercetin as standard $\left(0-50 \mathrm{mg} \mathrm{L}^{-1}\right)$ was used and the data was expressed as $\mathrm{mg}$ of quercetin equivalents (QE) per $\mathrm{g}$ of dry matter (DM).

\subsection{Determination of Total Monomeric Anthocyanin Content (TAC)}

TAC of extracts of the juçara waste was estimated by the $\mathrm{pH}$ differential method. In solution, at $\mathrm{pH} 1.0$ anthocyanins exhibit predominantly the colored oxonium form whereas, at $\mathrm{pH} 4.5$ there is a shift towards the colorless hemiketal form; therefore, it is possible to estimate TAC by the difference between absorbance values at $520 \mathrm{~nm}$ (Lee, Durst, \& Wrolstad, 2005). Absorbance values at 520 and $700 \mathrm{~nm}$ were evaluated using Spectrophotometer UV-1800 (Shimadzu Corporation, Kyoto, Japan). The wavelength of $700 \mathrm{~nm}$ was utilized to correct the influence of haze on sample absorbance. The TAC value was calculated as follows (Equation 2):

$$
T A C=\frac{A \times M W \times D F \times 10^{3}}{\varepsilon \times 1}
$$

Where, A equals the difference between (Abs $520 \mathrm{~nm}(\mathrm{pH} \mathrm{1.0)-Abs} 700 \mathrm{~nm}(\mathrm{pH} \mathrm{1.0)})$ and (Abs $520 \mathrm{~nm}(\mathrm{pH}$ 4.5)-Abs $700 \mathrm{~nm} \mathrm{pH}$ (4.5)); $\mathrm{MW}$ is the molecular weight $449.2 \mathrm{~g} \mathrm{~mol}^{-1}$ of cyanidin-3-glucoside (cyd-3-glu); DF is dilution factor of each sample; $10^{3}$ is the unit conversion from $\mathrm{g}$ to $\mathrm{mg} ; \varepsilon$ is 26,900 molar extinction coefficient, in $\mathrm{L} \mathrm{mol}{ }^{-1} \mathrm{~cm}^{-1}$, for cyd-3-glu; and 1 is light path length in $\mathrm{cm}$. The results were expressed as $\mathrm{mg}$ cyanidin-3-glucoside equivalents per $100 \mathrm{~g}$ of dry matter (DM). 
Table 1. Extraction conditions of the experimental design and results of total phenolic content (TPC), total flavonoids content (TFC), total monomeric anthocyanin content (TAC), total tannins content (TTC) and antioxidant activity (AA\%)

\begin{tabular}{|c|c|c|c|c|c|c|c|c|c|c|c|c|c|}
\hline \multirow{3}{*}{$\begin{array}{l}\text { Run } \\
\text { Order }\end{array}$} & \multicolumn{3}{|c|}{ Extraction conditions } & \multirow{2}{*}{\multicolumn{2}{|c|}{$\begin{array}{c}\text { TPC } \\
\left(\mathrm{mg} \mathrm{GAE}^{-}\right. \\
\left.100 \mathrm{~g} \mathrm{DM}^{-1}\right)\end{array}$}} & \multirow{2}{*}{\multicolumn{2}{|c|}{$\begin{array}{c}\text { TFC } \\
(\mathrm{mg} \mathrm{QE} \cdot \\
\left.\mathrm{g} \mathrm{DM}^{-1}\right)\end{array}$}} & \multirow{2}{*}{\multicolumn{2}{|c|}{$\begin{array}{c}\text { TAC } \\
(\mathrm{mg} \mathrm{C} \mathrm{QQE} \\
\left.100 \mathrm{~g} \mathrm{DM}^{-1}\right)\end{array}$}} & \multirow{2}{*}{\multicolumn{2}{|c|}{$\begin{array}{c}\text { TTC } \\
(\% \mathrm{mg} \mathrm{TAE} \\
\left.100 \mathrm{~g} \mathrm{DM}^{-1}\right)\end{array}$}} & \multirow{2}{*}{\multicolumn{2}{|c|}{$\mathbf{A A} \%_{120 \min }$}} \\
\hline & $\begin{array}{l}\text { Microwave } \\
\text { Powder }\end{array}$ & $\begin{array}{l}\text { Exposition } \\
\text { Time }\end{array}$ & $\begin{array}{l}\text { Ethanol } \\
\text { Concentration }\end{array}$ & & & & & & & & & & \\
\hline & (W) $X_{1}$ & (s) $\mathrm{X}_{2}$ & $(\%) X_{3}$ & Exp. & Pred. & Exp. & Pred. & Exp. & Pred. & Exp. & Pred. & $\overline{\operatorname{Exp}}$ & Pred \\
\hline$\# 1$ & $\begin{array}{l}668.18 \\
(1.68)\end{array}$ & $60(0)$ & $60(0)$ & $\begin{array}{l}1893.98 \\
\pm 33.11^{\mathrm{a}}\end{array}$ & $1905.60^{\mathrm{a}}$ & $\begin{array}{l}156.99 \\
\pm 1.26^{\mathrm{a}}\end{array}$ & $159.19^{\mathrm{a}}$ & $\begin{array}{l}249.06 \\
\pm 0.67^{\mathrm{a}}\end{array}$ & $235.32^{\mathrm{a}}$ & $\begin{array}{l}0.98 \\
\pm 0.06^{\mathrm{a}}\end{array}$ & $0.82^{\mathrm{a}}$ & $\begin{array}{l}61.56 \\
\pm 3.02^{\mathrm{a}}\end{array}$ & $64.14^{\mathrm{a}}$ \\
\hline$\# 2$ & $\begin{array}{l}331.82 \\
(-1.68)\end{array}$ & $60(0)$ & $60(0)$ & $\begin{array}{l}1948.53 \\
\pm 67.50^{\mathrm{a}}\end{array}$ & $1930.79^{\mathrm{a}}$ & $\begin{array}{l}163.23 \\
\pm 5.04^{\mathrm{a}}\end{array}$ & $162.29^{\mathrm{a}}$ & $\begin{array}{l}207.35 \\
\pm 0.94^{\mathrm{a}}\end{array}$ & $217.95^{\mathrm{a}}$ & $\begin{array}{l}0.50 \\
\pm 0.04^{\mathrm{a}}\end{array}$ & $0.44^{\mathrm{a}}$ & $\begin{array}{l}56.75 \\
\pm 3.83^{\mathrm{a}}\end{array}$ & $53.22^{\mathrm{a}}$ \\
\hline$\# \mathbf{3}$ & $500(0)$ & $60(0)$ & $60(0)$ & $\begin{array}{l}1281.74 \\
\pm 50.15^{\mathrm{a}}\end{array}$ & $1279.54^{\mathrm{a}}$ & $\begin{array}{l}148.07 \\
\pm 8.83^{\mathrm{a}}\end{array}$ & $160.74^{\mathrm{a}}$ & $\begin{array}{l}244.16 \\
\pm 1.98^{\mathrm{a}}\end{array}$ & $249.94^{\mathrm{a}}$ & $\begin{array}{l}0.63 \\
\pm 0.06^{\mathrm{a}}\end{array}$ & $0.63^{\mathrm{a}}$ & $\begin{array}{l}61.94 \\
\pm 1.57^{\mathrm{a}}\end{array}$ & $58.68^{\mathrm{a}}$ \\
\hline$\# 4$ & $500(0)$ & $60(0)$ & $60(0)$ & $\begin{array}{l}1298.43 \\
\pm 35.50^{\mathrm{a}}\end{array}$ & $1279.54^{\mathrm{a}}$ & $\begin{array}{l}160.96 \\
\pm 2.72^{\mathrm{a}}\end{array}$ & $160.74^{\mathrm{a}}$ & $\begin{array}{l}245.26 \\
\pm 3.35^{\mathrm{a}}\end{array}$ & $249.94^{\mathrm{a}}$ & $\begin{array}{l}0.66 \\
\pm 0.05^{\mathrm{a}}\end{array}$ & $0.63^{\mathrm{a}}$ & $\begin{array}{l}60.71 \\
\pm 3.58^{\mathrm{a}}\end{array}$ & $58.68^{\mathrm{a}}$ \\
\hline$\# 5$ & $600(1)$ & $90(1)$ & $80(1)$ & $\begin{array}{l}2171.34 \\
\pm 33.11^{b}\end{array}$ & $2223.79^{\mathrm{a}}$ & $\begin{array}{l}230.13 \\
\pm 3.78^{\mathrm{a}}\end{array}$ & $228.22^{\mathrm{a}}$ & $\begin{array}{l}350.58 \\
\pm 6.04^{\mathrm{a}}\end{array}$ & $355.10^{\mathrm{a}}$ & $\begin{array}{l}0.63 \\
\pm 0.05^{\mathrm{a}}\end{array}$ & $0.63^{\mathrm{a}}$ & $\begin{array}{l}65.85 \\
\pm 4.47^{\mathrm{a}}\end{array}$ & $67.07^{\mathrm{a}}$ \\
\hline$\# 6$ & $400(-1)$ & $90(1)$ & $40(-1)$ & $\begin{array}{l}2045.87 \\
\pm 52.09^{\mathrm{a}}\end{array}$ & $1859.79^{\mathrm{a}}$ & $\begin{array}{l}163.23 \\
\pm 0.00^{\mathrm{a}}\end{array}$ & $162.16^{\mathrm{a}}$ & $\begin{array}{l}187.67 \\
\pm 2.90^{\mathrm{a}}\end{array}$ & $182.27^{\mathrm{a}}$ & $\begin{array}{l}0.68 \\
\pm 0.03^{\mathrm{a}}\end{array}$ & $0.64^{\mathrm{a}}$ & $\begin{array}{l}63.27 \\
\pm 4.88^{\mathrm{a}}\end{array}$ & $60.57^{\mathrm{a}}$ \\
\hline$\# 7$ & $400(-1)$ & $30(-1)$ & $80(1)$ & $\begin{array}{l}968.84 \\
\pm 42.25^{\mathrm{a}}\end{array}$ & $948.78^{\mathrm{a}}$ & $\begin{array}{l}198.31 \\
\pm 1.03^{\mathrm{a}}\end{array}$ & $198.24^{\mathrm{a}}$ & $\begin{array}{l}259.02 \\
\pm 4.02^{\mathrm{a}}\end{array}$ & $258.61^{\mathrm{a}}$ & $\begin{array}{l}0.80 \\
\pm 0.01^{\mathrm{a}}\end{array}$ & $0.78^{\mathrm{a}}$ & $\begin{array}{l}51.65 \\
\pm 3.34^{\mathrm{a}}\end{array}$ & $50.30^{\mathrm{a}}$ \\
\hline$\# 8$ & $600(1)$ & $30(-1)$ & $40(-1)$ & $\begin{array}{l}939.43 \\
\pm 2.55^{\mathrm{a}}\end{array}$ & $988.99^{\mathrm{a}}$ & $\begin{array}{l}130.04 \\
\pm 3.56^{\mathrm{a}}\end{array}$ & $128.49^{\mathrm{a}}$ & $\begin{array}{l}230.56 \\
\pm 4.02^{\mathrm{a}}\end{array}$ & $239.11^{\mathrm{a}}$ & $\begin{array}{l}0.59 \\
\pm 0.06^{\mathrm{a}}\end{array}$ & $0.46^{\mathrm{a}}$ & $\begin{array}{l}57.49 \\
\pm 3.87^{\text {a }}\end{array}$ & $56.79^{\mathrm{a}}$ \\
\hline$\# 9$ & $500(0)$ & $60(0)$ & $60(0)$ & $\begin{array}{l}1185.57 \\
\pm 6.82^{\mathrm{a}}\end{array}$ & $1279.54^{\mathrm{a}}$ & $\begin{array}{l}157.88 \\
\pm 4.72^{\mathrm{a}}\end{array}$ & $160.74^{\mathrm{a}}$ & $\begin{array}{l}250.48 \\
\pm 5.70^{\mathrm{a}}\end{array}$ & $249.94^{\mathrm{a}}$ & $\begin{array}{l}0.62 \\
\pm 0.05^{\mathrm{a}}\end{array}$ & $0.62^{\mathrm{a}}$ & $\begin{array}{l}54.93 \\
\pm 2.84^{\mathrm{a}}\end{array}$ & $58.68^{\mathrm{a}}$ \\
\hline$\# 10$ & $500(0)$ & $60(0)$ & $60(0)$ & $\begin{array}{l}1209.58 \\
\pm 39.83^{\mathrm{a}}\end{array}$ & $1279.54^{\mathrm{a}}$ & $\begin{array}{l}153.12 \\
\pm 2,06^{\mathrm{a}}\end{array}$ & $160.74^{\mathrm{a}}$ & $\begin{array}{l}248.59 \\
\pm 3.29^{\mathrm{a}}\end{array}$ & $249.94^{\mathrm{a}}$ & $\begin{array}{l}0.62 \\
\pm 0.03^{\mathrm{a}}\end{array}$ & $0.62^{\mathrm{a}}$ & $\begin{array}{l}59.90 \\
\pm 3.34^{\mathrm{a}}\end{array}$ & $58.68^{\mathrm{a}}$ \\
\hline \#11 & $500(0)$ & $60(0)$ & $\begin{array}{l}93.64 \\
(1.68)\end{array}$ & $\begin{array}{l}1486.94 \\
\pm 68.77^{\mathrm{a}}\end{array}$ & $1415.68^{\mathrm{a}}$ & $\begin{array}{l}251.24 \\
\pm 10.45^{\mathrm{a}}\end{array}$ & $245.19^{\mathrm{a}}$ & $\begin{array}{l}351.06 \\
\pm 3.29^{\mathrm{a}}\end{array}$ & $349.49^{\mathrm{a}}$ & $\begin{array}{l}0.76 \\
\pm 0.04^{\mathrm{a}}\end{array}$ & $0.75^{\mathrm{a}}$ & $\begin{array}{l}60.06 \\
\pm 0.52^{\mathrm{a}}\end{array}$ & $58.68^{\mathrm{a}}$ \\
\hline$\# 12$ & $500(0)$ & $60(0)$ & $\begin{array}{l}26.37 \\
(-1.68)\end{array}$ & $\begin{array}{l}920.21 \\
\pm 14.56^{\mathrm{a}}\end{array}$ & $1143.00^{\mathrm{a}}$ & $\begin{array}{l}174.53 \\
\pm 1.03^{\mathrm{a}}\end{array}$ & $181.12^{\mathrm{a}}$ & $\begin{array}{l}182.32 \\
\pm 4.03^{\mathrm{a}}\end{array}$ & $187.76^{\mathrm{a}}$ & $\begin{array}{l}0.47 \\
\pm 0.03^{\mathrm{a}}\end{array}$ & $0.50^{\mathrm{a}}$ & $\begin{array}{l}56.32 \\
\pm 2.21^{\mathrm{a}}\end{array}$ & $58.68^{\mathrm{a}}$ \\
\hline$\# 13$ & $400(-1)$ & $90(1)$ & $80(1)$ & $\begin{array}{l}1411.30 \\
\pm 12.61^{\mathrm{a}}\end{array}$ & $1622.92^{\mathrm{a}}$ & $\begin{array}{l}197.12 \\
\pm 3.09^{\mathrm{a}}\end{array}$ & $200.25^{\mathrm{a}}$ & $\begin{array}{l}354.38 \\
\pm 2.01^{\mathrm{a}}\end{array}$ & $344.77^{\mathrm{a}}$ & $\begin{array}{l}0.45 \\
\pm 0.05^{\mathrm{a}}\end{array}$ & $0.41^{\mathrm{a}}$ & $\begin{array}{l}61.40 \\
\pm 3.68^{\mathrm{a}}\end{array}$ & $60.57^{\mathrm{a}}$ \\
\hline \#14 & $500(0)$ & $60(0)$ & $60(0)$ & $\begin{array}{l}1195.17 \\
\pm 8.25^{\mathrm{a}}\end{array}$ & $1079.19^{\mathrm{a}}$ & $\begin{array}{l}151.93 \\
\pm 2,72^{\mathrm{a}}\end{array}$ & $160.74^{\mathrm{a}}$ & $\begin{array}{l}253.33 \\
\pm 4.03^{\mathrm{a}}\end{array}$ & $249.94^{\mathrm{a}}$ & $\begin{array}{l}0.57 \\
\pm 0.05^{\mathrm{a}}\end{array}$ & $0.62^{\mathrm{a}}$ & $\begin{array}{l}65.69 \\
\pm 3.71^{\mathrm{a}}\end{array}$ & $58.68^{\mathrm{a}}$ \\
\hline \#15 & $500(0)$ & $\begin{array}{l}110.45 \\
(1.68)\end{array}$ & $60(0)$ & $\begin{array}{l}1589.60 \\
\pm 17.83^{\mathrm{a}}\end{array}$ & $1529.59^{\mathrm{a}}$ & $\begin{array}{l}193.56 \\
\pm 3.57^{\mathrm{a}}\end{array}$ & $187.50^{\mathrm{a}}$ & $\begin{array}{l}287.49 \\
\pm 4.03^{\mathrm{a}}\end{array}$ & $296.21^{\mathrm{a}}$ & $\begin{array}{l}0.68 \\
\pm 0.15^{\mathrm{a}}\end{array}$ & $0.65^{\mathrm{a}}$ & $\begin{array}{l}70.72 \\
\pm 2.98^{\mathrm{a}}\end{array}$ & $67.32^{\mathrm{a}}$ \\
\hline$\# 16$ & $600(1)$ & $90(1)$ & $40(-1)$ & $\begin{array}{l}1456.93 \\
\pm 41.44^{\mathrm{a}}\end{array}$ & $1522.67^{\mathrm{a}}$ & $\begin{array}{l}188.80 \\
\pm 1.03^{\mathrm{a}}\end{array}$ & $190.12^{\mathrm{a}}$ & $\begin{array}{l}190.71 \\
\pm 4.02^{\mathrm{a}}\end{array}$ & $192.60^{\mathrm{a}}$ & $\begin{array}{l}0.88 \\
\pm 0.06^{\mathrm{a}}\end{array}$ & $0.87^{\mathrm{a}}$ & $\begin{array}{l}62.21 \\
\pm 0.82^{\mathrm{a}}\end{array}$ & $67.07^{\mathrm{a}}$ \\
\hline$\# 17$ & $500(0)$ & $60(0)$ & $60(0)$ & $\begin{array}{l}1315.24 \\
\pm 53.74^{\mathrm{a}}\end{array}$ & $1079.19^{\mathrm{a}}$ & $\begin{array}{l}173.34 \\
\pm 2.72^{\mathrm{a}}\end{array}$ & $160.74^{\mathrm{a}}$ & $\begin{array}{l}259.02 \\
\pm 4.02^{\mathrm{a}}\end{array}$ & $249.94^{\mathrm{a}}$ & $\begin{array}{l}0.55 \\
\pm 0.10^{\mathrm{a}}\end{array}$ & $0.62^{\mathrm{a}}$ & $\begin{array}{l}64.11 \\
\pm 0.34^{\mathrm{a}}\end{array}$ & $58.68^{\mathrm{a}}$ \\
\hline \#18 & $400(-1)$ & $30(-1)$ & $40(-1)$ & $\begin{array}{l}932.82 \\
\pm 20.87^{\mathrm{a}}\end{array}$ & $1050.89^{\mathrm{a}}$ & $\begin{array}{l}160.26 \\
\pm 2.72^{\mathrm{a}}\end{array}$ & $160.14^{\mathrm{a}}$ & $\begin{array}{l}241.94 \\
\pm 4.02^{\mathrm{a}}\end{array}$ & $228.78^{\mathrm{a}}$ & $\begin{array}{l}0.17 \\
\pm 0.04^{\mathrm{a}}\end{array}$ & $0.23^{\mathrm{a}}$ & $\begin{array}{l}51.66 \\
\pm 3.35^{\text {a }}\end{array}$ & $50.30^{\mathrm{a}}$ \\
\hline$\# 19$ & $600(1)$ & $30(-1)$ & $80(1)$ & $\begin{array}{l}1184.37 \\
\pm 48.39^{\mathrm{a}}\end{array}$ & $1023.49^{\mathrm{a}}$ & $\begin{array}{l}157.88 \\
\pm 1.78^{\mathrm{a}}\end{array}$ & $166.59^{\mathrm{a}}$ & $\begin{array}{l}264.72 \\
\pm 4.02^{\mathrm{a}}\end{array}$ & $268.93^{\mathrm{a}}$ & $\begin{array}{l}0.40 \\
\pm 0.07^{\mathrm{b}}\end{array}$ & $1.00^{\mathrm{a}}$ & $\begin{array}{l}58.67 \\
\pm 2.61^{\mathrm{a}}\end{array}$ & $56.79^{\mathrm{a}}$ \\
\hline$\# 20$ & $500(0)$ & $\begin{array}{l}9.55 \\
(-1.68)\end{array}$ & $60(0)$ & $\begin{array}{l}595.43 \\
\pm 22.57^{\mathrm{a}}\end{array}$ & $628.80^{\mathrm{a}}$ & $\begin{array}{l}137.36 \\
\pm 1.26^{\mathrm{a}}\end{array}$ & $133.98^{\mathrm{a}}$ & $\begin{array}{l}264.72 \\
\pm 4.03^{\mathrm{a}}\end{array}$ & $262.86^{\mathrm{a}}$ & $\begin{array}{l}0.57 \\
\pm 0.12^{\mathrm{a}}\end{array}$ & $0.61^{\mathrm{a}}$ & $\begin{array}{l}48.77 \\
\pm 2.38^{\mathrm{a}}\end{array}$ & $50.04^{\mathrm{a}}$ \\
\hline
\end{tabular}

Note. All results are the means $\pm \mathrm{SD}(\mathrm{n}=3)$.

${ }^{a-b}$ Same letters prescribe that there was no difference between the experimental and predicted results within the analysis; different letters determine the difference.

Exp.: Experimental results; Pred.: Predicted results; W: watts; s: seconds.

\subsection{Determination of Total Tannin Content (TTC)}

TAC of the E. edulis waste extract was estimated according to the methodology of Makkar (2003). This method is based on the precipitation of condensed tannins using PVPP. In the first step was measured the content of total phenolics at the absorbance value at $725 \mathrm{~nm}$ using Spectrophotometer UV-1800 (Shimadzu Corporation, Kyoto, Japan). Whereas for the second step, were precipitated tannins and the absorbance value of the decantate was evaluated at $725 \mathrm{~nm}$ using Spectrophotometer UV-1800 (Shimadzu Corporation, Kyoto, Japan). The difference between the absorbance values between the first and the second steps was utilized to estimate the TTC value 
through a calibration curve of tannic acid as standard $\left(0-14 \mu \mathrm{g} \mathrm{mL}^{-1}\right)$ and expressed per $100 \mathrm{~g}$ of dry matter (DM).

\subsection{Antioxidant Activity}

$\beta$-carotene bleaching assay was used to determine the antioxidant activity of extracts by the $\beta$-carotene-linoleic acid model system (Siraichi et al., 2013) with modifications. Briefly, $1 \mathrm{~mL}$ of $\beta$-carotene $(0.2 \mathrm{mg} / \mathrm{mL}$ ) was pipetted into a glass tube with $20 \mathrm{~mL}$ of linoleic acid, $200 \mathrm{mg}$ of Tween 40 . The chloroform was completely evaporated by using a rotary evaporator (QUIMIS, Brazil). After, $50 \mathrm{~mL}$ of distilled water were added to the flask with vigorous stirring. Another emulsion was made without $\beta$-carotene. Aliquots $(4.8 \mathrm{~mL})$ of the prepared emulsions were transferred to a series of tubes containing $0.2 \mathrm{~mL}$ of extracts. The tubes were placed in a water bath at $50{ }^{\circ} \mathrm{C}$ for $2 \mathrm{~h}$.

The absorbance of each sample was measured using a Spectrophotometer UV-1800 (Shimadzu Corporation, Kyoto, Japan) set at 470 and $700 \mathrm{~nm}$ immediately after sample preparation $(\mathrm{t}=0 \mathrm{~min})$ and at 30 -min intervals until the end $(t=120 \mathrm{~min})$ of the experiment; the latter wavelength was utilized to correct the influence of haze. Emulsion without $\beta$-carotene using like blank. Water and BHT $(3 \mathrm{mg} / \mathrm{mL})$ were used as negative and positive controls, respectively. The antioxidant activity of extracts was expressed as (Equation 3):

$$
100 \times \frac{\left(1-\left(\left(A_{0}-A_{c 0}\right)-\left(A_{t}-A_{c t}\right)\right)\right)}{\left(\left(\left(A_{w 0}-A_{w c 0}\right)-\left(A_{w t}-A_{w c t}\right)\right)+\left(\left(A_{B H T 0}-A_{B H T c 0}\right)-\left(A_{B H T t}-A_{B H T c t}\right)\right)\right)}
$$

\subsection{Statistical Analysis}

All analyses are carried out in triplicate and results reported as mean values with standard deviation. ANOVA with Turkey test was performed using XLSTAT version 2013.2.03 (Addinsoft, Paris, France). RSM was performed using the Minitab ${ }^{\circledR}$ software (version 17.1.0, USA). The regression coefficients of linear, square and two-way interaction terms were evaluated by analysis of variance, and the relevant $(p<0.05)$ terms were utilized to generate the surface and contour plots. The fitted polynomial equation indicated the optimal conditions for the TPC, TFC, TAC and TTC response variables. Differences in phenolic, flavonoids, monomeric anthocyanin and tannins compounds were considered significant at $p<0.05$., you may refer the reader to that source and simply give a brief synopsis of the method in this section.

\section{Results and Discussion}

\subsection{Antioxidant Compounds Extraction}

For antioxidant compounds extraction, ethanol aqueous solution is a preferred solvent system (Karacabey \& Mazza, 2010; Sharma et al., 2014), since polyphenols have a varied range of solubility (Ilaiyaraja, Likhith, Sharath Babu, \& Khanum, 2015), and use of solvents with different polarity potentials high yield of total antioxidants (Szydłowska-Czerniak, Tułodziecka, Karlovits, \& Szłyk, 2015). Ethanol, a green safe solvent, (Kukula-Koch et al., 2013; Li et al., 2012) was utilized due to efficiency on solubilizing bioactive molecules from vegetable matrixes (Espinosa-Pardo et al., 2014; Fang, Wang, Hao, Li, \& Guo, 2015; Sharma et al., 2014). In addition, previous studies using MAE to the extraction of antioxidant compounds from fruits determined that the concentration of the solvent, microwave power and exposition time were independents variables (Dahmoune et al., 2014, 2015; Fang et al., 2015; Kim et al., 2012; Li et al., 2012, 2014; Zeković et al., 2016).

In the present study, the response data of TPC, TFC, TAC, TTC and AA\% were listed in Table 1. The highest TPC was represented for experiment \#5 (2171.34 mg GAE·100 g DM$\left.{ }^{-1}\right)$ that correspond to microwave power 600 (W), ethanol concentration $80 \%$ and time 90 seconds provided. For TFC, the experiment \#11 (500 W, $93.46 \%$ and 60 seconds) showed the highest concentration (251.24 mg QE.g DM ${ }^{-1}$ ), while in TAC was the experiment \#13 (400 W, ethanol concentration $80 \%$ and 90 seconds) (354.38 mg C3QE·100 g DM ${ }^{-1}$ ). As for TTC the experiment \#1 (668.18 W, ethanol concentration $60 \%$ and 60 seconds) exhibited the highest tannins concentration (0.98 mg TAE $100 \mathrm{~g} \mathrm{DM}^{-1}$ ). Regarding AA\%, the experiment \#15 (500 W, ethanol concentration $60 \%$ and $110.45 \mathrm{sec})$ obtained the highest antioxidant activity $(70.72 \%)$.

The RSM indicated the empirical relationship between TPC (Equation 4), TFC (Equation 5), TAC (Equation 6), TTC (Equation 7) and AA\% (Equation 8) value with the extraction conditions were generated as follows:

$$
\begin{gathered}
Y=6298-22.65 X_{1}+7.03 X_{2}-6.81 X_{3}+0.02258 X_{1}^{2}+0.1809 X_{2} X_{3} \\
Y=392.2-0.3073 X_{1}-1.953 X_{2}-4.607 X_{3}+0.04633 X_{3}^{2}+0.004968 X_{1} X_{2} \\
Y=154.3+0.876 X_{1}-4.381 X_{2}-2.894 X_{3}-0.000824 X_{1}^{2}+0.01162 X_{2}^{2}+0.01651 X_{3}^{2}+0.05528 X_{2} X_{3} \\
Y=-1.355+0.001142 X_{1}+0.01972 X_{2}+0.02323 X_{3}-0.000323 X_{2} X_{3}
\end{gathered}
$$




$$
Y=32.57+0.0325 X_{1}+0.1712 X_{2}
$$

Moreover, ANOVA for experimental results show the quadratic polynomial model for TPC, TFC, TAC, TTC and AA\% was significant highly $(\mathrm{F}=30.78, \mathrm{~F}=46.03, \mathrm{~F}=50.81, \mathrm{~F}=9.01, \mathrm{~F}=8.89$, respectively), with $\mathrm{p}<0.001$ for TPC, TFC and TAC; $p=0.001$ for TTC; $p=0.002$ for AA\%, (Table 2). There is only a 0.01 to $0.002 \%$ chance that a "Model F-Value" this large could occur due to noise, recommended the significant of the model. $\mathrm{R}^{2}$ and $\mathrm{R}^{2}$ adjusted (Table 2) values for the model did not differ considerably, this confirms an adequate statistical model. However, a large value of $\mathrm{R}^{2}$ not necessarily designates that the regression model is a sound one. Therefore, it is better to use the $\mathrm{R}^{2}$ adjusted to evaluate the model adequacy, as that the addition of a variable to the model $\mathrm{R}^{2}$ increase with the significant or non-significant variable (Karazhiyan, Razavi, \& Phillips, 2011). The absence of lack of fit and the value of pure error indicated good reproducibility of the experimental data (Table 2). The model could work well for the prediction of TPC, TFC, TAC, TTC and AA\% extract from $E$. edulis waste powder.

Table 2. Analysis of variance (ANOVA) and regression coefficients for the quadratic polynomial model for the experimental results of total phenolic (TPC), total flavonoids (TFC), total monomeric anthocyanin (TAC), total tannins content (TTC) and antioxidant activity (AA\%) from Euterpe edulis waste extract

\begin{tabular}{|c|c|c|c|c|c|c|c|c|c|c|c|c|c|c|c|}
\hline \multirow[t]{2}{*}{ Analysis } & & \multirow{2}{*}{ Model } & \multirow{2}{*}{$\begin{array}{l}\text { Intercept } \\
\text { B0 }\end{array}$} & \multicolumn{3}{|c|}{ Linear } & \multicolumn{3}{|c|}{ Square } & \multicolumn{2}{|c|}{$\begin{array}{c}\text { 2-Way } \\
\text { Interaction }\end{array}$} & \multirow{2}{*}{$\begin{array}{l}\text { Lack } \\
\text { of fit }\end{array}$} & \multirow{2}{*}{$\begin{array}{l}\text { Pure } \\
\text { error }\end{array}$} & \multirow[t]{2}{*}{ Residual } & \multirow{2}{*}{$\begin{array}{l}\text { Corr. } \\
\text { Total }\end{array}$} \\
\hline & & & & $\overline{X_{1}}$ & $X_{2}$ & $X_{3}$ & $\overline{X_{1}^{1}}$ & $X_{2}^{2}$ & $X_{3}^{3}$ & $X_{1} X_{2}$ & $X_{2} X_{3}$ & & & & \\
\hline $\begin{array}{l}\text { TPC }^{\mathrm{b}} \\
\mathrm{R}^{2}=0.9166\end{array}$ & $\begin{array}{l}\text { Standard } \\
\text { error }\end{array}$ & 50 & 50 & 38.6 & 44 & 5.09 & & 37 & & & 6.91 & & & & \\
\hline $\mathrm{R}_{\mathrm{A}}^{2}=0.8869$ & $\mathrm{DF}^{\mathrm{a}}$ & 5 & & 1 & 1 & 1 & & 1 & & & 1 & 10 & 4 & 14 & 19 \\
\hline \multirow[t]{3}{*}{$\mathrm{R}_{\mathrm{P}}^{2}=0.8581$} & $\begin{array}{l}\text { Sum of } \\
\text { squares }\end{array}$ & 3026928 & 2050435 & 4016 & 1639587 & 406831 & & & & & 245352 & 259124 & 16194 & 275318 & 3302247 \\
\hline & F-value & 30.78 & 16.80 & 0.04 & 37.04 & 12.79 & & 37.18 & & & 12.48 & 6.90 & & & \\
\hline & P-value & $<0.001$ & $<0.001$ & 0.849 & $<0.001$ & 0.003 & & $<0.001$ & & & 0.003 & 0.154 & & & \\
\hline $\begin{array}{l}\mathrm{TFC}^{\mathrm{c}} \\
\mathrm{R}^{2}=0.9427\end{array}$ & $\begin{array}{l}\text { Standard } \\
\text { error }\end{array}$ & 12.90 & 12.90 & 2.20 & 2.20 & 2.42 & & 0.108 & & 2.88 & & & & & \\
\hline $\mathrm{R}_{\mathrm{A}}^{2}=0.9222$ & $\mathrm{DF}^{\mathrm{a}}$ & 5 & & 1 & 1 & 1 & & 1 & & 1 & & 9 & 5 & 14 & 19 \\
\hline \multirow[t]{3}{*}{$\mathrm{R}_{\mathrm{P}}^{2}=0.8859$} & $\begin{array}{l}\text { Sum of } \\
\text { squares }\end{array}$ & 15240.2 & 8423.9 & 11.6 & 3457.6 & 4954.8 & & 5039.4 & & 1776.9 & & 373.4 & 553.7 & 927.1 & 320972 \\
\hline & F-value & 46.03 & 32.72 & 0.18 & 52.21 & 45.77 & & 76.10 & & 26.83 & & 0.37 & & & \\
\hline & P-value & $<0.001$ & $<0.001$ & 0.682 & $<0.001$ & $<0.001$ & & $<0.001$ & & $<0.001$ & & 0.905 & & & \\
\hline $\mathrm{R}^{2}=0.9674$ & $\begin{array}{l}\text { Standard } \\
\text { error }\end{array}$ & 18.00 & 18.00 & 3.06 & 10.40 & 3.39 & 2.98 & 2.98 & 0.15 & & 0.90 & & & & \\
\hline $\mathrm{R}_{\mathrm{A}}^{2}=0.9483$ & $\mathrm{DF}^{\mathrm{a}}$ & 7 & & 1 & 1 & 1 & 1 & 1 & 1 & & 1 & 7 & 5 & 12 & 19 \\
\hline \multirow[t]{3}{*}{$\mathrm{R}_{\mathrm{P}}^{2}=0.8507$} & $\begin{array}{l}\text { Sum of } \\
\text { squares }\end{array}$ & 45511.4 & 33278.2 & 364.1 & 1342.2 & 31571.0 & 1404.0 & 1399.5 & 628.5 & & 8801.2 & 1384.2 & 151.2 & 1535.4 & 47046.7 \\
\hline & F-value & 50.81 & 17.47 & 2.85 & 48.53 & 1.04 & 8.94 & 7.65 & 12.33 & & 4.91 & 68.79 & & & \\
\hline & P-value & $<0.001$ & $<0.001$ & 0.017 & $<0.001$ & 0.327 & 0.017 & 0.004 & 0.047 & & $<0.001$ & 0.007 & & & \\
\hline $\begin{array}{l}\mathrm{TTC}^{\mathrm{e}} \\
\mathrm{R}^{2}=0.7510\end{array}$ & $\begin{array}{l}\text { Standard } \\
\text { error }\end{array}$ & 0.0881 & 0.0881 & 0.0337 & 0.0114 & 0.00759 & & & & & 0.0092 & & & & \\
\hline $\mathrm{R}_{\mathrm{A}}^{2}=0.6621$ & $\mathrm{DF}^{\mathrm{a}}$ & 4 & & 1 & 1 & 1 & & & & & 1 & 10 & 5 & 15 & 19 \\
\hline \multirow[t]{3}{*}{$\mathrm{R}_{\mathrm{P}}^{2}=0.5482$} & $\begin{array}{l}\text { Sum of } \\
\text { squares }\end{array}$ & 0.56068 & & 0.17820 & 0.00131 & 0.08039 & & & & & 0.30077 & 0.11979 & 0.11349 & & 0.79397 \\
\hline & F-value & 9.01 & 11.67 & 11.46 & 18.38 & 5.17 & & & & & 19.34 & 0.53 & & & \\
\hline & P-value & 0.001 & $<0.001$ & 0.004 & 0.001 & 0.038 & & & & & 0.001 & 0.818 & & & \\
\hline $\begin{array}{l}\mathrm{AA} \% \\
\mathrm{R}^{2}=0.5113\end{array}$ & $\begin{array}{l}\text { Standard } \\
\text { error }\end{array}$ & 1.19 & 1.19 & 1.14 & 1.14 & & & & & & & & & & \\
\hline $\mathrm{R}_{\mathrm{A}}^{2}=0.6538$ & $\mathrm{DF}^{\mathrm{a}}$ & 2 & 2 & 1 & 1 & & & & & & & 12 & 5 & & 19 \\
\hline \multirow[t]{3}{*}{$\mathrm{R}_{\mathrm{P}}^{2}=0.5860$} & $\begin{array}{l}\text { Sum of } \\
\text { squares }\end{array}$ & 504.4 & 504.4 & 144.0 & 360.4 & & & & & & & 286.1 & 196.0 & & 986.5 \\
\hline & F-value & 8.89 & 8.89 & 5.08 & 12.71 & & & & & & & 0.61 & & & \\
\hline & P-value & 0.002 & 0.002 & 0.038 & 0.002 & & & & & & & 0.778 & & & \\
\hline
\end{tabular}

Note. ${ }^{\mathrm{a}}$ Degree of freedom; ${ }^{\mathrm{b}}\left(\mathrm{mg} \mathrm{GAE} \cdot 100 \mathrm{~g} \mathrm{DM}^{-1}\right) ;{ }^{\mathrm{c}}\left(\mathrm{mg} \mathrm{QE} \cdot \mathrm{g} \mathrm{DM}^{-1}\right) ;{ }^{\mathrm{d}}\left(\mathrm{mg} \mathrm{C} 3 \mathrm{QE} \cdot 100 \mathrm{~g} \mathrm{DM}^{-1}\right) ;{ }^{\mathrm{e}}(\mathrm{mg} \mathrm{TAE} \cdot 100 \mathrm{~g}$ $\left.\mathrm{DM}^{-1}\right) ; \mathrm{R}_{\mathrm{A}}^{2}=\mathrm{R}^{2}$ Adjusted; $\mathrm{R}_{\mathrm{P}}^{2}=\mathrm{R}^{2}$ Predicted; $X_{1}=$ Power $(\mathrm{W}) ; X_{2}=$ Time (s); $X_{3}=$ Ethanol Concentration (\%). 
The experimentally optimized conditions described by the model to selected for maximum TPC and TFC extraction is microwave power $668.18 \mathrm{~W}$, exposition time of 110.45 seconds and aqueous ethanol concentration 93.64\%. For maximum TAC extraction is microwave power $532.28 \mathrm{~W}$, exposition time of 110.45 seconds and aqueous ethanol concentration $93.64 \%$. For TTC extraction is microwave power $668.18 \mathrm{~W}$, exposition time of 9.55 seconds and aqueous ethanol concentration 93.64\%. To obtain the optimum maximum extraction of antioxidants molecules studied, the parameter was determined: $668.18 \mathrm{~W}, 110.45$ seconds and $93.64 \%$ by the model. And for antioxidant activity is microwave power $668.18 \mathrm{~W}$, exposition time of 110.45 seconds and aqueous ethanol $64.41 \%$.

\subsection{Interactions of the Studied Factors}

The interaction between microwave power, exposition time and ethanol aqueous solution between TPC are shown in three-dimensional surface and contour plots (Figure 1A). The contour plots indicate the nature and extent of interactions of different components (Prakash, Talat, Hasan, \& Pandey, 2008). The maximum point of each three-dimension plot subjected is the optimum point for the two factors presented in the chart. The effect between the exposition time $\left(X_{2}\right)$ and ethanol concentration $\left(X_{3}\right)$ are presented in Figure 1-A1, this can be observed the time and ethanol concentration is directly proportional to TPC yield, same behavior was observed in juçara pulp MAE (Cavalcanti et al., 2011), in coriander seed extracts (Zekovi et al., 2016), in Cammelia oleifera fruit (Zhang et al., 2011) and in Vitis coignetiae (Kim et al., 2012). Thus, the highest ethanol concentration increased extraction of compounds (Kim et al., 2012), this may be attributed the difference in dielectric properties of solvent towards microwave heating (Dahmoune et al., 2014).

The effect between the ratio of microwave power $\left(X_{1}\right)$ and ethanol concentration $\left(X_{3}\right)$ are represented in Figure 1-A2. First, the extraction combination decreased efficiency by raise power but after $550 \mathrm{~W}$ increased. Probably because increased diffusion rate and solubility of the target compounds in the solvent were affected by temperature (Fernández-Ponce, Casas, Mantell, \& Martínez de la Ossa, 2015) and higher power must be excited phenolic molecules (Zeković et al., 2016). Also, an increase of ethanol concentration increase TPC yield, probably for the fact of solvent polarity declined and solubility of molecules increased. The same behavior has observed in Pistacia lentiscus and Vitis coignetiae (Dahmoune et al., 2014; Kim et al., 2012). This behavior was observed for phenolic compounds from Euterpe edulis peels and pulp near the seeds by Garcia-Mendonza et al. (Garcia-Mendoza et al., 2017). According to Fernández-Ponce et al. (2015) and Santos et al. (2012), others fruits extracts presented the same behaviors for temperature action. The interaction of ratio of microwave power $\left(X_{1}\right)$ and exposition time $\left(X_{2}\right)$ are presented in Figure 1-A3. The yield of TPC decreases with the increase of power, probably due to thermal degradation of phenolic compounds (Dahmoune et al., 2015; Dairi et al., 2015). 

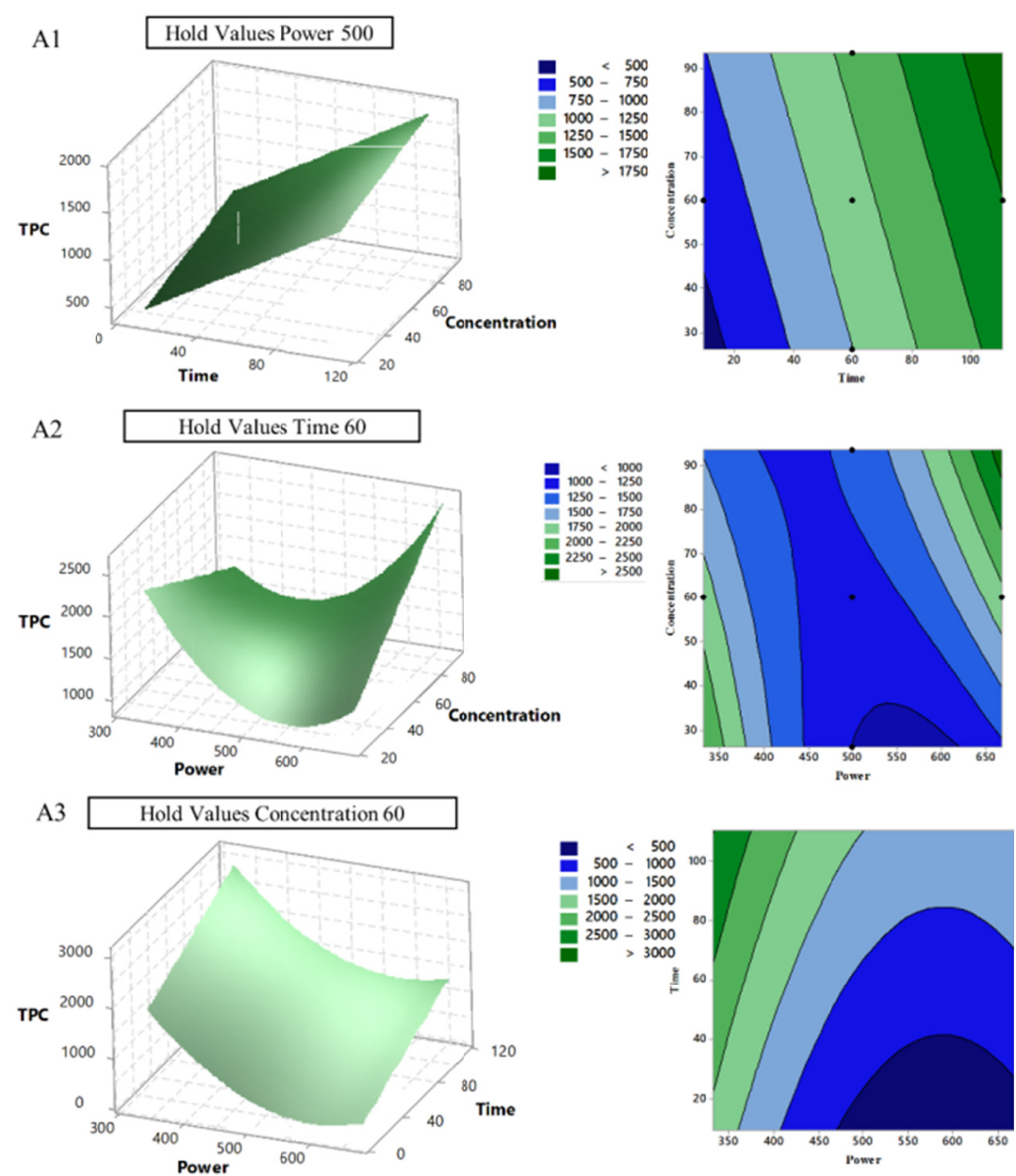

Figure 1A. Response surface plots showing the operating parameter 2-Way Interaction on total phenolic (TPC). The surface and contour plots describing the effect of three independent variables on response variables; TPC interaction between variables $X_{1}$ to $X_{3}$ (A1-A3). TPC: mg GAE $100 \mathrm{~g} \mathrm{DM}^{-1}$, Microwave power (W), exposition time (seconds) and ethanol concentration in aqueous solution as solvent (\%)

Referring to the interaction between studied factors with TFC (Figure 1B). Interaction of concentration and time (Figure 1-B1) present decrease yield in about $50 \%$ of ethanol. The same behavior was observed in the interaction of power and ethanol concentration (Figure 1-B2), this can be explained by the fact of flavonoids represent a wide range of polarity and ethanol presents a molecule with apolar and polar activity (Fattahi \& Rahimi, 2016). In Figure 1-B3 increase power about $500 \mathrm{~W}$ and exposition time of 40 seconds can be attributed the highest yield of TFC, it can be explained by the fact of after $500 \mathrm{~W}$ the temperature can be high them $55^{\circ} \mathrm{C}$ and flavonoids molecules are degraded in temperatures higher than $55^{\circ} \mathrm{C}$ (Fattahi \& Rahimi, 2016). 

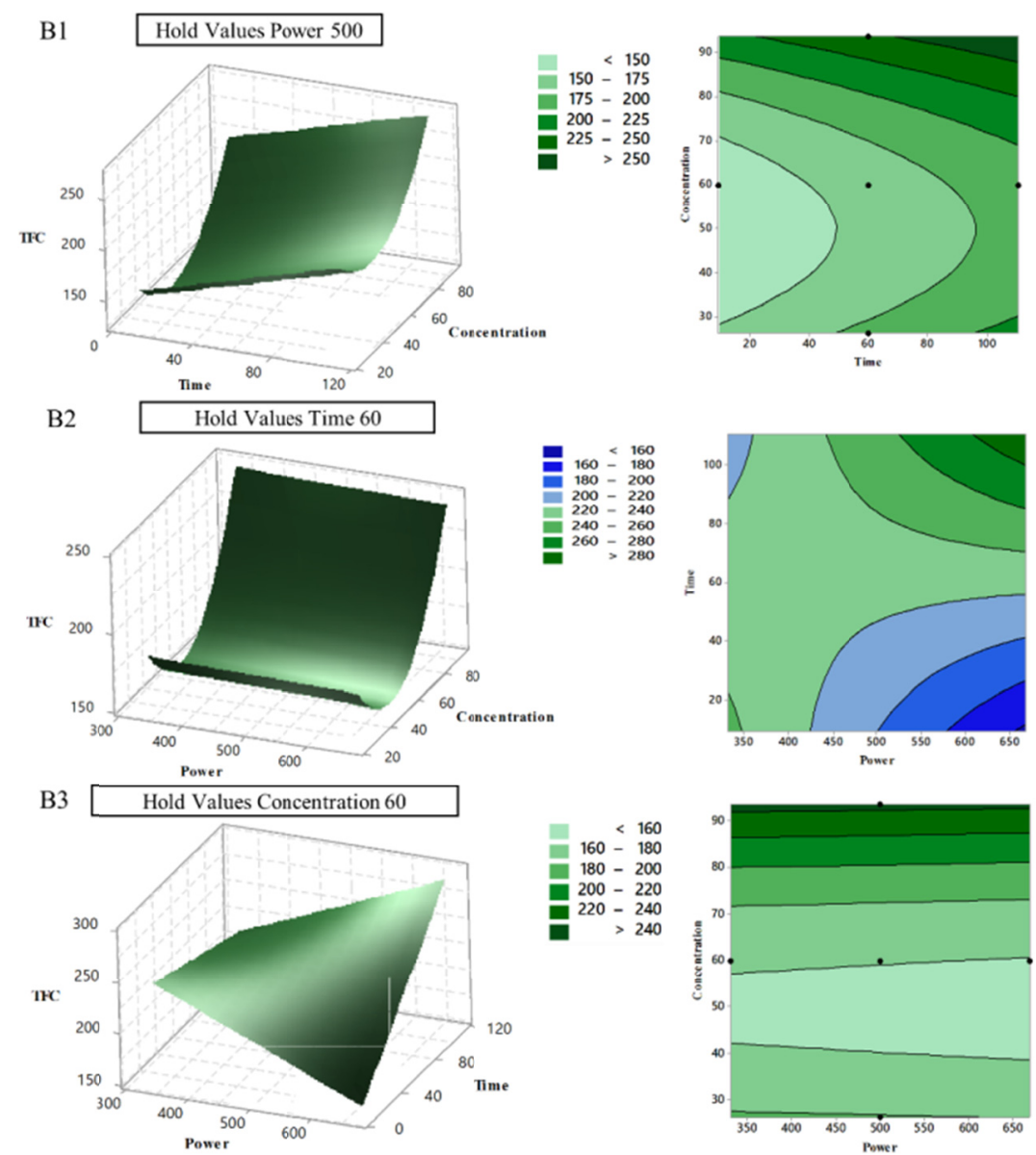

Figure 1B. Response surface plots showing the operating parameter 2-Way Interaction flavonoids (TFC). The surface and contour plots describing the effect of three independent variables on response variables TFC interaction between variables $X_{1}$ to $X_{3}$ (B1-B3); TFC: $\mathrm{mg} \mathrm{QE} \cdot \mathrm{g} \mathrm{DM}^{-1}$, Microwave power (W), exposition time (seconds) and ethanol concentration in aqueous solution as solvent (\%)

The interaction between studied factors on TAC extraction is represented in three-dimensional surface and contour plots (Figure 2A). The interaction of power and exposition time show monomeric anthocyanins decrease (Figure 2-A1), possible, for the fact of the increase in temperature and molecules degradation (Dahmoune et al., 2015; Dairi et al., 2015). Ethanol concentration and microwave power interaction presented in Figure 2-A2 demonstrated that initially with to increase of power, the yield of TAC increased, however about 500 to $560 \mathrm{~W}$ its began to decrease. This fact can be explaining the fact of a possibility of high temperature and degradation of monomeric anthocyanins (Jiménez et al., 2010). When the waste of E. edulis exposed more time, and increase an ethanol concentration the yield of TAC increased, this can be explained by the fact of anthocyanins represent a wide range of polarity, and ethanol presents a molecule with apolar and polar activity (Fattahi \& Rahimi, 2016). Similarly interaction behavior was observed in antioxidant compounds by Kim et al. (2012) between microwave power and exposition time in Figure 2-A3 show the TFC yield decrease with time and power increase, this can be explained by high temperature and degradation of monomeric anthocyanins (Jiménez et al., 2010). 

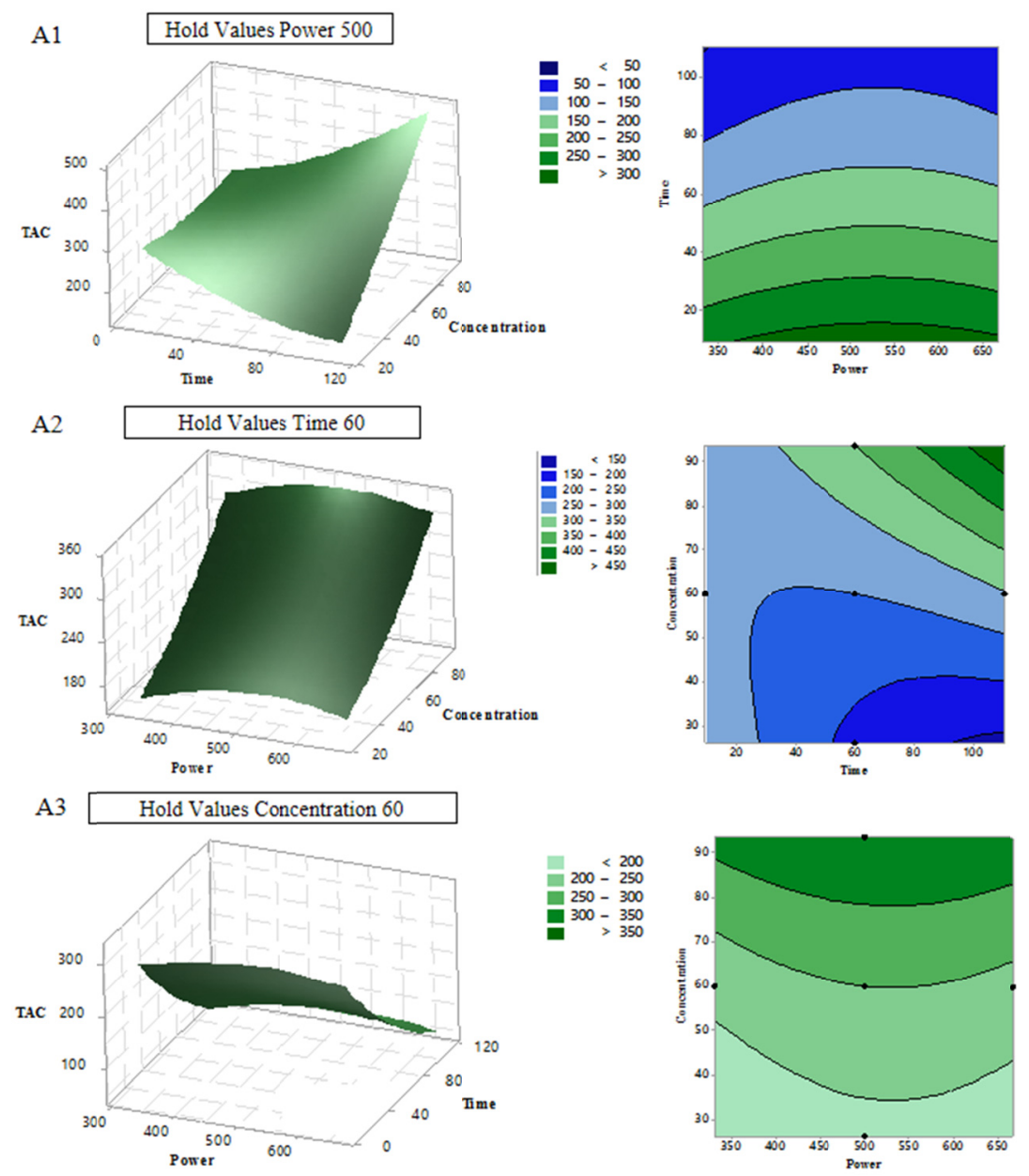

Figure 2A. Response surface plots showing the operating parameter 2-Way Interaction anthocyanins (TAC). The surface and contour plots describing the effect of three independent variables on response variables; TAC interaction between variables $X_{1}$ to $X_{3}$ (A1-A3). TAC: $\mathrm{mg} \mathrm{C} 3 \mathrm{QE} \cdot 100 \mathrm{~g} \mathrm{DM}^{-1}$, Microwave power (W), exposition time (seconds) and ethanol concentration in aqueous solution as solvent (\%)

Three-dimensional surface plots and contour plots for TTC based interaction between studied factors are shown in Figure 2B. In the interaction of exposition time and ethanol aqueous concentration (Figure 2-B1), the highest yield obtained with low time, about 6 to 10 seconds, and up $45 \%$ of ethanol has also an increase, but after 45 to $50 \%$ had decreased, can be explained by the fact of ethanol be less polar than water (Szydłowska-Czerniak et al., 2015). In Figure 2-B2, TTC increased with the interaction of concentration and microwave power. When observed power and exposition time interaction (Figure 2-B3), the yield increased with time at about $550 \mathrm{~W}$. Parada, Rodríguez-Blanco, Fernández de Ana Magán, and Domínguez (2015) observed the highest extraction of antioxidant compounds about 450 to $550 \mathrm{~W}$, similar to found in this study. 

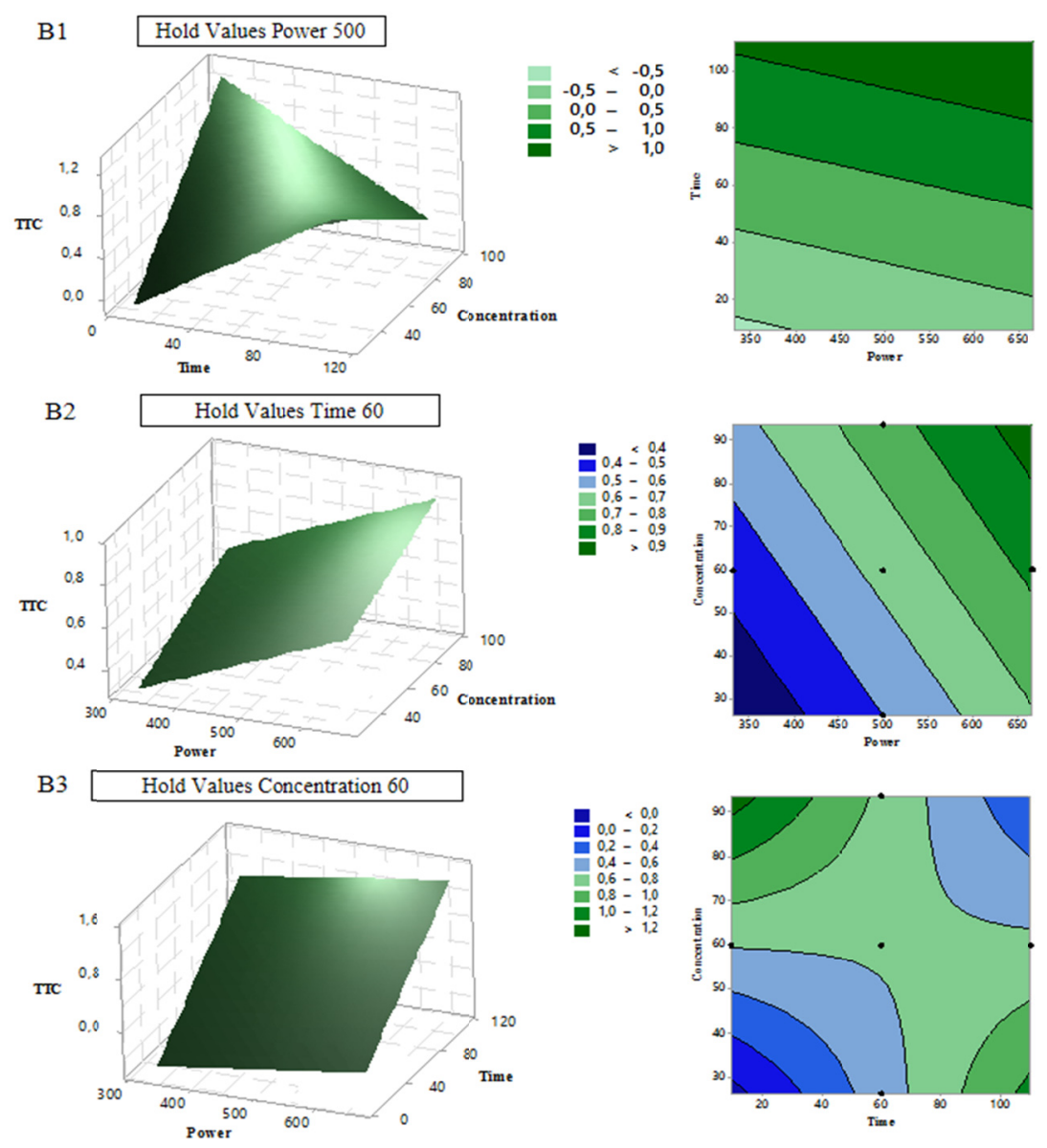

Figure 2B. Response surface plots showing the operating parameter 2-Way Interaction tannins (TTC) content. The surface and contour plots describing the effect of three independent variables on response variables; TTC interaction between variables $X_{1}$ to $X_{3}$ (B1-B3). TTC: mg TAE·100 g DM-1 , Microwave power (W), exposition time (seconds) and ethanol concentration in aqueous solution as solvent (\%)

The interaction of variables and extraction of potential antioxidant activity extract were present in three-dimensional and contour plots (Figure 3A). Only microwave power and exposition time can be affected obtain of extraction with high antioxidant activity. This can be explained by the fact that compounds extract with different ethanol concentrations balanced this function since antioxidant activity depends on the type of antioxidants in the extract and not the quantity (Fattahi \& Rahimi, 2016). The power increased can promote an increase of temperature during extraction, so increase the phenolic extraction, this occurs because the higher rate of mass transfer at high temperature, which would have dissolved the phenolic compounds more easily (Li et al., 2012). The same behavior can be observed in other fruits as tomatoes ( $\mathrm{Li}$ et al., 2012).

A

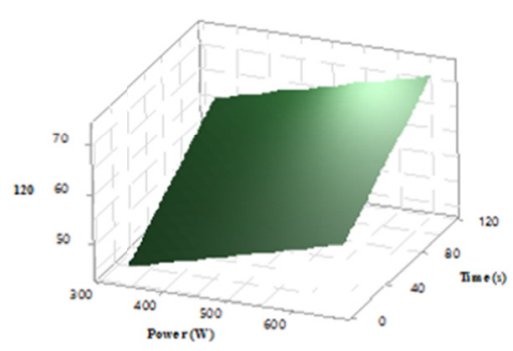

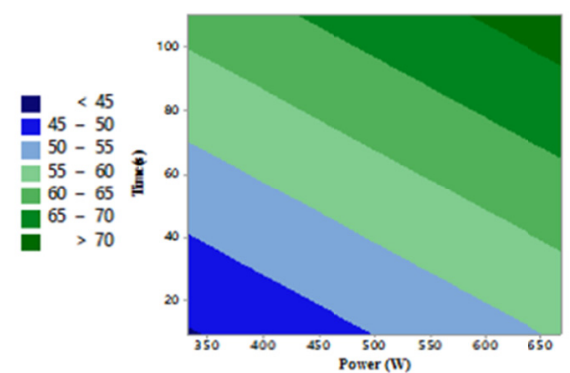

Figure 3A. Response surface plots showing the operating parameter 2-way interaction antioxidant activity, the surface and contour plots describing the effect of three independent variables on response variables 
The antioxidant extracts were similar behavior, as shown in Figure 3B, and grouped within in close absorbance. For determining antioxidant activity, water was used as a negative control, when to compare obtained extracts to water activity and BHT (positive control), E. edulis waste extracts presented close behavior of BHT (Figure 3B). For this fact, these extracts can be compared to BHT antioxidant activity, so E. edulis waste is a potential source of natural antioxidants.

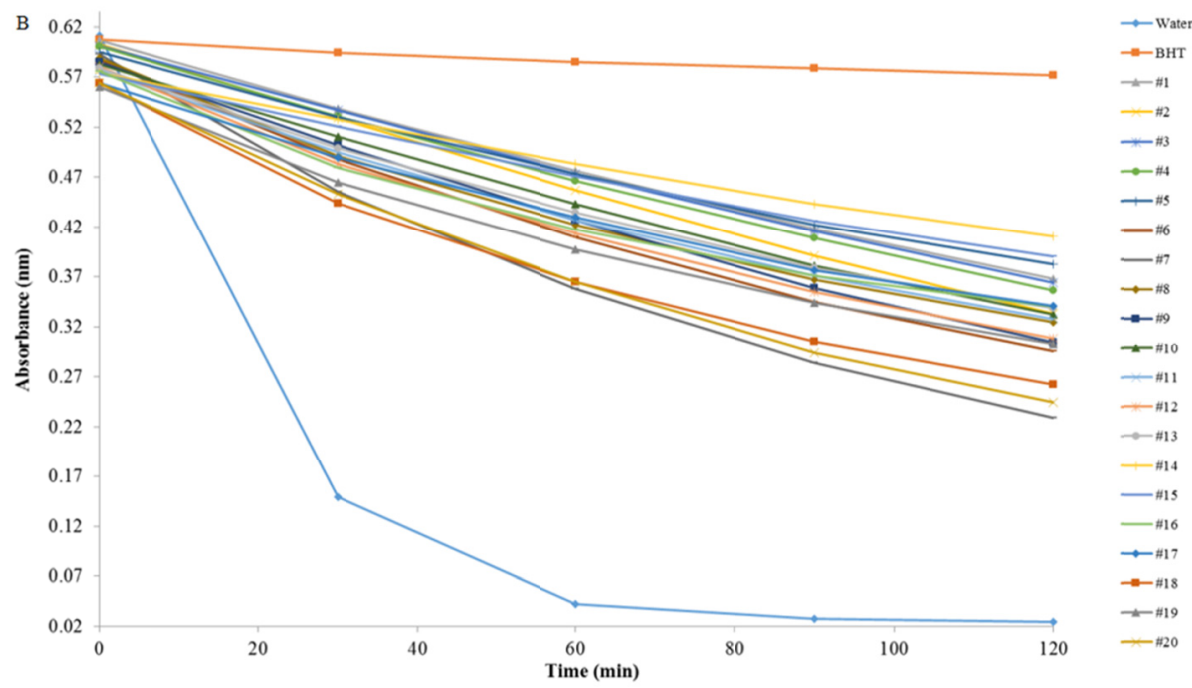

Figure 3B. Antioxidant activity of ethanolic extracts from E. edulis, as assessed by the coupled oxidation of $\beta$-carotene and linoleic acid over $120 \mathrm{~min}$

\subsection{Validation and Verification of Predictive Models}

To validate the predicted data (from the equations generated by the models obtained) must be compared to the experimental results (Table 1) by ANOVA and Turkey test. In addition, it was observed that there was no difference between the results obtained in the experiment and predicted by the model. Hence, we can confirm as an optimal condition to obtain the compounds contents and the antioxidant capacity of the extract by microwave extraction using power, exposition time and ethanol concentration in the solvent as variables. However, future studies should be performed to evaluate the effect of these extracts in the food matrix, which would indicate if it is appropriate to use them industrially. Nevertheless, the parameters evaluated in this study can be used to predict the concentration of total phenolic, flavonoids, anthocyanins and tannins present in the extracts of E. edulis waste, with high antioxidant activity, including study characteristics and individual effect sizes used in a meta-analysis, can be made available on supplemental online archives.

\section{Conclusion}

The extraction performance was influenced by microwave power, exposition time and ethanol aqueous solution concentration. In addition, the experimental design was successfully applied for optimization of high antioxidant activity extracts. Thus, MAE conditions were well optimized for the extraction of antioxidant compounds from $E$. edulis wastes. The optimal conditions that maximized the extraction yields of antioxidant compounds in Euterpe edulis wastes were microwave power at $668.17 \mathrm{~W}$, ethanol concentration at $93.65 \%$ and 65.60 seconds, and of antioxidant activity were microwave power at $668.17 \mathrm{~W}$, ethanol concentration at $64.41 \%$ and 110.45 seconds.

\section{References}

Ainsworth, E. A., \& Gillespie, K. M. (2007). Estimation of total phenolic content and other oxidation substrates in plant tissues using Folin-Ciocalteu reagent. Nature Protocols, 2(4), 875-877. https://oi.org/10.1038/ nprot.2007.102

Bicudo, M. O. P., Ribani, R. H., \& Beta, T. (2014). Anthocyanins, Phenolic Acids and Antioxidant Properties of Juçara Fruits (Euterpe edulis M.) Along the On-tree Ripening Process. Plant Foods for Human Nutrition, 69(2), 142-147. https://doi.org/10.1007/s11130-014-0406-0 
Borges, G. D. S. C., Gonzaga, L. V., Jardini, F. A., Mancini Filho, J., Heller, M., Micke, G., ... Fett, R. (2013). Protective effect of Euterpe edulis M. on Vero cell culture and antioxidant evaluation based on phenolic composition using HPLC-ESI-MS/MS. Food Research International, 51(1), 363-369. https://doi.org/ 10.1016/j.foodres.2012.12.035

Borges, G., Gracieli Kunradi Vieira, F., Copetti, C., Valdemiro Gonzaga, L., Zambiazi, R. C., Mancini Filho, J., \& Fett, R. (2011). Chemical characterization, bioactive compounds, and antioxidant capacity of jussara (Euterpe edulis) fruit from the Atlantic Forest in southern Brazil. Food Research International, 44(7), 2128-2133. https://doi.org/10.1016/j.foodres.2010.12.006

Cavalcanti, R. N., Santos, D. T., \& Meireles, M. A. A. (2011). Non-thermal stabilization mechanisms of anthocyanins in model and food systems-An overview. Food Research International, 44(2), 499-509. https://doi.org/10.1016/j.foodres.2010.12.007

Chang, C.-C., Yang, M.-H., Wen, H.-M., \& Chern, J.-C. (2002). Estimation of total flavonoid content in propolis by two complementary colorimetric methods. Journal of Food and Drug Analysis, 10(3). Retrieved from http://search.proquest.com/openview/8ed5f78aa3317908039c7a8ca3740050/1?pq-origsite=gscholar

Contini, C., Álvarez, R., O’Sullivan, M., Dowling, D. P., Gargan, S. Ó., \& Monahan, F. J. (2014). Effect of an active packaging with citrus extract on lipid oxidation and sensory quality of cooked turkey meat. Meat Science, 96(3), 1171-1176. https://doi.org/10.1016/j.meatsci.2013.11.007

Dahmoune, F., Nayak, B., Moussi, K., Remini, H., \& Madani, K. (2015). Optimization of microwave-assisted extraction of polyphenols from Myrtus communis L. leaves. Food Chemistry, 166, 585-595. https://doi.org/10.1016/j.foodchem.2014.06.066

Dahmoune, F., Spigno, G., Moussi, K., Remini, H., Cherbal, A., \& Madani, K. (2014). Pistacia lentiscus leaves as a source of phenolic compounds: Microwave-assisted extraction optimized and compared with ultrasound-assisted and conventional solvent extraction. Industrial Crops and Products, 61, 31-40. https://doi.org/10.1016/j.indcrop.2014.06.035

Dairi, S., Galeano-Díaz, T., Acedo-Valenzuela, M. I., Godoy-Caballero, M. P., Dahmoune, F., Remini, H., \& Madani, K. (2015). Monitoring oxidative stability and phenolic compounds composition of myrtle-enriched extra virgin olive during heating treatment by flame, oven and microwave using reversed phase dispersive liquid-liquid microextraction (RP-DLLME)-HPLC-DAD-FLD method. Industrial Crops and Products, 65, 303-314. https://doi.org/10.1016/j.indcrop.2014.11.035

Ertas, A., Boga, M., Yilmaz, M. A., Yesil, Y., Tel, G., Temel, H., Ugurlu, P. (2015). A detailed study on the chemical and biological profiles of essential oil and methanol extract of Thymus nummularius (Anzer tea): Rosmarinic acid. Industrial Crops and Products, 67, 336-345. https://doi.org/10.1016/j.indcrop.2015.01.064

Espinosa-Pardo, F. A., Martinez, J., \& Martinez-Correa, H. A. (2014). Extraction of bioactive compounds from peach palm pulp (Bactris gasipaes) using supercritical $\mathrm{CO}_{2}$. The Journal of Supercritical Fluids, 93, 2-6. https://doi.org/10.1016/j.supflu.2014.05.010

Fang, X., Wang, J., Hao, J., Li, X., \& Guo, N. (2015). Simultaneous extraction, identification and quantification of phenolic compounds in Eclipta prostrata using microwave-assisted extraction combined with HPLC-DAD-ESI-MS/MS. Food Chemistry, 188, 527-536. https://doi.org/10.1016/j.foodchem.2015.05.037

Fattahi, M., \& Rahimi, R. (2016). Optimization of Extraction Parameters of Phenolic Antioxidants from Leaves of Capparis spinosa Using Response Surface Methodology. Food Analytical Methods, 9(8), 2321-2334. https://doi.org/10.1007/s12161-016-0414-9

Fernández-Ponce, M. T., Casas, L., Mantell, C., \& Martínez de la Ossa, E. (2015). Use of high pressure techniques to produce Mangifera indica L. leaf extracts enriched in potent antioxidant phenolic compounds. Innovative Food Science \& Emerging Technologies, 29, 94-106. https://doi.org/10.1016/j.ifset.2015.04.006

Garcia-Mendoza, M. del P., Espinosa-Pardo, F. A., Baseggio, A. M., Barbero, G. F., Maróstica Junior, M. R., Rostagno, M. A., \& Martínez, J. (2017). Extraction of phenolic compounds and anthocyanins from juçara (Euterpe edulis Mart.) residues using pressurized liquids and supercritical fluids. The Journal of Supercritical Fluids, 119, 9-16. https://doi.org/10.1016/j.supflu.2016.08.014

Ilaiyaraja, N., Likhith, K. R., Sharath Babu, G. R., \& Khanum, F. (2015). Optimisation of extraction of bioactive compounds from Feronia limonia (wood apple) fruit using response surface methodology (RSM). Food Chemistry, 173, 348-354. https://doi.org/10.1016/j.foodchem.2014.10.035 
Jiménez, N., Bohuon, P., Lima, J., Dornier, M., Vaillant, F., \& Pérez, A. M. (2010). Kinetics of Anthocyanin Degradation and Browning in Reconstituted Blackberry Juice Treated at High Temperatures $\left(100-180{ }^{\circ} \mathrm{C}\right)$. Journal of Agricultural and Food Chemistry, 58(4), 2314-2322. https://doi.org/10.1021/jf902381e

Karacabey, E., \& Mazza, G. (2010). Optimisation of antioxidant activity of grape cane extracts using response surface methodology. Food Chemistry, 119(1), 343-348. https://doi.org/10.1016/j.foodchem.2009.06.029

Karazhiyan, H., Razavi, S. M. A., \& Phillips, G. O. (2011). Extraction optimization of a hydrocolloid extract from cress seed (Lepidium sativum) using response surface methodology. Food Hydrocolloids, 25(5), 915-920. https://doi.org/10.1016/j.foodhyd.2010.08.022

Kim, H.-K., Do, J.-R., Lim, T.-S., Akram, K., Yoon, S.-R., \& Kwon, J.-H. (2012). Optimisation of microwave-assisted extraction for functional properties of Vitis coignetiae extract by response surface methodology. Journal of the Science of Food and Agriculture, 92(8), 1780-1785. https://doi.org/10.1002/ jsfa.5546

Kukula-Koch, W., Aligiannis, N., Halabalaki, M., Skaltsounis, A.-L., Glowniak, K., \& Kalpoutzakis, E. (2013). Influence of extraction procedures on phenolic content and antioxidant activity of Cretan barberry herb. Food Chemistry, 138(1), 406-413. https://doi.org/10.1016/j.foodchem.2012.10.045

Lee, J., Durst, R. W., \& Wrolstad, R. E. (2005). Determination of total monomeric anthocyanin pigment content of fruit juices, beverages, natural colorants, and wines by the $\mathrm{pH}$ differential method: Collaborative study. Journal of AOAC International, 88(5), 1269-1278.

Li, H., Deng, Z., Wu, T., Liu, R., Loewen, S., \& Tsao, R. (2012). Microwave-assisted extraction of phenolics with maximal antioxidant activities in tomatoes. Food Chemistry, 130(4), 928-936. https://doi.org/10.1016/ j.foodchem.2011.08.019

Li, M., Ngadi, M. O., \& Ma, Y. (2014). Optimisation of pulsed ultrasonic and microwave-assisted extraction for curcuminoids by response surface methodology and kinetic study. Food Chemistry, 165, 29-34. https://doi.org/10.1016/j.foodchem.2014.03.115

Li, Y., Fabiano-Tixier, A.-S., Abert-Vian, M., \& Chemat, F. (2013). Microwave-assisted Extraction for Bioactive Compounds: Theory and practice. In F. Chemat \& G. Cravotoo (Eds.), Microwave-Assisted Extraction of Antioxidants and Food Colors (Chapter 5). Food Engineering Series. New York Heidelberg Dordrecht London: Springer.

Lu, J., Qin, P., Han, X., Wang, Y., \& Li, Z. (2015). Evaluation of antioxidant and antibacterial properties of extracts from Trollius chinensis Bunge. European Food Research and Technology, 240(2), 301-310. https://doi.org/10.1007/s00217-014-2329-2

Makkar, H. P. (2003). Quantification of tannins in tree and shrub foliage: A laboratory manual (p. 20). Springer Science \& Business Media. Kluwer Academic Publishers.

Manganaris, G. A., Goulas, V., Vicente, A. R., \& Terry, L. A. (2014). Berry antioxidants: Small fruits providing large benefits. Journal of the Science of Food and Agriculture, 94(5), 825-833. https://doi.org/10.1002/ jsfa. 6432

Montgomery, D. C. (2004). Design and analysis of experiments. John Wiley. http://bcs.wiley.com/he-bcs/ Books?action=index\&itemId=047148735X\&itemTypeId=BKS\&bcsId $=2172$

Ortega-Ramirez, L. A., Rodriguez-Garcia, I., Leyva, J. M., Cruz-Valenzuela, M. R., Silva-Espinoza, B. A., Gonzalez-Aguilar, G. A., \& Ayala-Zavala, J. F. (2014). Potential of Medicinal Plants as Antimicrobial and Antioxidant Agents in Food Industry: A Hypothesis: Medicinal plants as food additives. Journal of Food Science, 79(2), R129-R137. https://doi.org/10.1111/1750-3841.12341

Parada, M., Rodríguez-Blanco, A., Fernández de Ana Magán, F., \& Domínguez, H. (2015). Sequential extraction of Hericium erinaceus using green solvents. LWT - Food Science and Technology, 64(1), 397-404. https://doi.org/10.1016/j.lwt.2015.06.008

Prakash, O., Talat, M., Hasan, S. H., \& Pandey, R. K. (2008). Factorial design for the optimization of enzymatic detection of cadmium in aqueous solution using immobilized urease from vegetable waste. Bioresource Technology, 99(16), 7565-7572. https://doi.org/10.1016/j.biortech.2008.02.008

Rahal, A., Kumar, A., Singh, V., Yadav, B., Tiwari, R., Chakraborty, S., \& Dhama, K. (2014). Oxidative Stress, Prooxidants, and Antioxidants: The Interplay. BioMed Research International, 1-19. https://doi.org/10.1155/ $2014 / 761264$ 
Rufino, M. S. M., Alves, R. E., Fernandes, F. A. N., \& Brito, E. S. (2011). Free radical scavenging behavior of ten exotic tropical fruits extracts. Food Research International, 44(7), 2072-2075. https://doi.org/10.1016/ j.foodres.2010.07.002

Santos, D. T., Veggi, P. C., \& Meireles, M. A. A. (2010). Extraction of antioxidant compounds from Jabuticaba (Myrciaria cauliflora) skins: Yield, composition and economical evaluation. Journal of Food Engineering, 101(1), 23-31. https://doi.org/10.1016/j.jfoodeng.2010.06.005

Santos, D. T., Veggi, P. C., \& Meireles, M. A. A. (2012). Optimization and economic evaluation of pressurized liquid extraction of phenolic compounds from jabuticaba skins. Journal of Food Engineering, 108(3), 444-452. https://doi.org/10.1016/j.jfoodeng.2011.08.022

Shao, Y., Wu, Q., Duan, J., Yue, W., Gu, W., \& Wang, X. (2014). Optimisation of the solvent extraction of bioactive compounds from Lophatherum gracile Brongn. using response surface methodology and HPLC-PAD coupled with pre-column antioxidant assay. Anal. Methods, 6(1), 170-177. https://doi.org/ 10.1039/C3AY41279B

Sharma, K., Assefa, A. D., Kim, S., Ko, E. Y., Lee, E. T., \& Park, S. W. (2014). Evaluation of total phenolics, flavonoids and antioxidant activity of 18 Korean onion cultivars: A comparative study. Journal of the Science of Food and Agriculture, 94(8), 1521-1529. https://doi.org/10.1002/jsfa.6450

Silva, P. P. M., do CARMO, L. F., Silva, G. M., Silveira-Diniz, M. F., Casemiro, R. C., \& Spoto, M. H. F. (2013). Physical, chemical, and lipid composition of juçara (Euterpe edulis Mart.) pulp. Brazilian Journal of Food and Nutrition, 24(1), 7-13.

Siraichi, J. T. G., Felipe, D. F., Brambilla, L. Z. S., Gatto, M. J., Terra, V. A., Cecchini, A. L., ... Cortez, D. A. G. (2013). Antioxidant Capacity of the Leaf Extract Obtained from Arrabidaea chica Cultivated in Southern Brazil. PLoS ONE, 8(8), e72733. https://doi.org/10.1371/journal.pone.0072733

Szydłowska-Czerniak, A., Tułodziecka, A., Karlovits, G., \& Szłyk, E. (2015). Optimisation of ultrasound-assisted extraction of natural antioxidants from mustard seed cultivars. Journal of the Science of Food and Agriculture, 95(7), 1445-1453. https://doi.org/10.1002/jsfa.6840

Tadapaneni, R. K., Daryaei, H., Krishnamurthy, K., Edirisinghe, I., \& Burton-Freeman, B. M. (2014). High-Pressure Processing of Berry and Other Fruit Products: Implications for Bioactive Compounds and Food Safety. Journal of Agricultural and Food Chemistry, 62(18), 3877-3885. https://doi.org/10.1021/ jf404400q

Zeković, Z., Vladić, J., Vidović, S., Adamović, D., \& Pavlić, B. (2016). Optimization of microwave-assisted extraction (MAE) of coriander phenolic antioxidants-response surface methodology approach: Microwave-assisted extraction of coriander phenolic antioxidants. Journal of the Science of Food and Agriculture, 96(13), 4613-4622. https://doi.org/10.1002/jsfa.7679

Zengin, G., Sarikurkcu, C., Aktumsek, A., \& Ceylan, R. (2014). Sideritis galatica Bornm.: A source of multifunctional agents for the management of oxidative damage, Alzheimer's's and diabetes mellitus. Journal of Functional Foods, 11, 538-547. https://doi.org/10.1016/j.jff.2014.08.011

Zhang, L., Wang, Y., Wu, D., Xu, M., \& Chen, J. (2011). Microwave-Assisted Extraction of Polyphenols from Camellia oleifera Fruit Hull. Molecules, 16(12), 4428-4437. https://doi.org/10.3390/molecules16064428

\section{Copyrights}

Copyright for this article is retained by the author(s), with first publication rights granted to the journal.

This is an open-access article distributed under the terms and conditions of the Creative Commons Attribution license (http://creativecommons.org/licenses/by/4.0/). 